\title{
THE POTENTIAL DISTRIBUTION IN A CONSTRICTED CYLINDER: AN EXACT SOLUTION*
}

BY

\author{
A. M. ROSENFELD AND R. S. TIMSIT
}

Aluminum Company of Canada, Ltd, Research and Development Centre, Kingston, Ontario

\begin{abstract}
An exact solution to the Laplace equation is derived for the distribution of electric potential within a long cylinder carrying a circular constriction along its axis. The expression obtained for the potential distribution is reduced to a form which may be readily evaluated and is highly accurate for a ratio of constriction radius to cylinder radius approaching unity. Exact expressions both for the electric current density within the constriction and for the spreading resistance (i.e., the increase in resistance of the cylinder due to constriction of current flow lines) are also obtained.
\end{abstract}

1. Introduction. The problem of determining the distribution of a vector flow field (an electric current density, for example) within a cylinder carrying a circular constriction along its axis finds application in various areas of fluid dynamics and electromagnetic theory and has been a subject of particular interest to several authors involved in the study of electrical and thermal contact resistance [1-4]. An exact solution to the problem has been derived [5] only in the limit as the ratio of the constriction radius $b$ to the cylinder radius $a$ approaches zero, i.e., for the particular situation of a constriction in an infinite body. The more interesting case when this ratio is an appreciable fraction of unity has heretofore been treated only approximately $[1,2,4]$. The difficulty with this situation is that it involves mixed boundary conditions which cannot be handled by conventional techniques of solution. The purpose of the present contribution is to show how these boundary conditions may be treated rigorously so that an expression, which is valid for all values of $b / a$, can be derived for the potential distribution in a constricted cylinder.

The approximate nature of the solutions obtained previously $[1,2,4]$ relates to the nature of the approximations introduced in dealing with the two boundary conditions in the plane of the constriction. These conditions require that the component of the electric current density normal to that plane vanish beyond the constriction edge and that the electric potential within the constriction be constant. The appproximation common to all previous treatments is a relaxation of these two boundary conditions in favor of the assumption of a specific current density distribution in the constriction. This formulation has the significant advantage of dispensing with the necessity of deriving this distribution from first principles. However, the form of the current density must be judiciously chosen so that violations of the boundary conditions stated above are minimized. Smythe [1], for example, chooses the current density in the constriction empirically to optimize coincidence

* Received June 4, 1980. 
of the zero equipotential surface with the constriction. In the solution obtained this coincidence is only approximately achieved but excursions of this equipotential from the plane of the constriction are minimal for relatively small values of $b / a$; the approach leads to satisfactory results only for $b / a \lesssim 0.5$. In another calculation [4] Timsit uses the known expression for the current density distribution corresponding to the limit $b / a \rightarrow 0$ and chooses a constant term in the solution again to minimize the variations of the potential within the constriction. This procedure leads to a solution capable of high numerical accuracy but only for $b / a \leqq 0.4$.

Clearly any formulation of the problem in terms of relaxed boundary conditions cannot lead to a satisfactory solution for all values of $b / a$. Such a solution must proceed from a rigorous treatment of all boundary conditions. The calculation presented in this paper follows such a treatment; no assumptions regarding the form of the current density distribution in the constriction are introduced. It is shown that the boundary conditions in the plane of the constriction lead to a system of dual Dini series equations which can be solved by a method due to Sneddon and Srivastav [6]. This procedure reduces the problem of solving simultaneously the dual series equations to a problem of solving a Fredholm integral equation of the second kind. The solution of this equation is used to generate the expression for the potential distribution. It is further shown that this expression may be simplified to a form involving a dominant term descriptive of the potential in the limit when $b / a \rightarrow 0$ and a sum of correction terms describing the perturbing effect of the finite radial extent of the cylinder on the current flow lines near the constriction. In this form, the solution may be readily evaluated numerically and is highly accurate for values of $b / a$ approaching unity.

2. Derivation of the solution. Following standard techniques in potential theory, the required axially symmetric solution of the Laplace equation

$$
\nabla^{2} V(r, z)=0
$$

for the distribution $V(r, z)$ of electric potential within the long constricted cylinder shown in Fig. 1 is given by

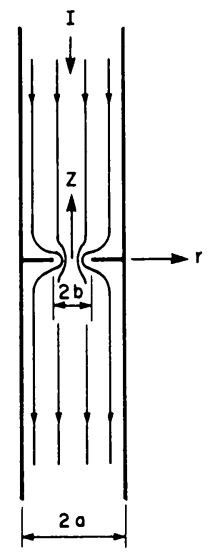

Fig. 1. 1. Schematic of the constricted cylinder. 


$$
V(r, z)=A+B z+\sum_{n=1}^{\infty} a_{n} k_{n}^{-1} J_{0}\left(k_{n} r\right) \exp \left(-k_{n} z\right)
$$

in the half-space $z \geq 0$. The quantities $A$ and $B$ are constants and $J_{0}(x)$ is the regular Bessel function of zero order; for an electrically insulated cylinder the quantities $k_{n}$ satisfy the relation $k_{n} a=\beta_{n}$ where $\beta_{n}$ is the $n$th positive zero of $J_{1}(x)$. In the present calculation the zero equipotential surface is defined to coincide with the constriction so that the potential distribution in the lower half-space is determined by the symmetry condition $V(r, z)=$ $-V(r,-z)$. The mixed boundary conditions in the plane of the constriction are given by

$$
\begin{gathered}
V(r, 0)=0, \quad 0 \leq r<b, \\
\frac{\partial}{\partial z} V(r, z)=0, \quad b<r \leq a, \quad z=0 .
\end{gathered}
$$

In view of expression (2) these conditions lead to the system of dual Dini series equations [7]

$$
\begin{gathered}
A+\sum_{n=1}^{\infty} a_{n} k_{n}^{-1} J_{0}\left(k_{n} r\right)=0, \quad 0 \leq r<b, \\
B-\sum_{n=1}^{\infty} a_{n} J_{0}\left(k_{n} r\right)=0, \quad b<r \leq a .
\end{gathered}
$$

The object of the present analysis is to derive a solution to the simultaneous equations (5) and (6) for the constant $B$ and coefficients $a_{n}$. The constant $A$ represents a scaling factor and is determined by a normalization condition.

At any point in the conductor the electric current density $\mathbf{J}(r, z)$ satisfies Ohm's law, $\mathbf{J}(r, z)=-\rho^{-1} \nabla V(r, z)$ where $\rho$ is the sample resistivity. It follows from Eq. (2) that the current density $j(r)=-J_{z}(r, 0)$ within the constriction is represented by the Dini series

$$
j(r)=\rho^{-1}\left[B-\sum_{n=1}^{\infty} a_{n} J_{0}\left(k_{n} r\right)\right], \quad 0 \leq r \leq a .
$$

This current density vanishes beyond the constriction edge $r=b$ in accordance with Eq. (6). It is clear from Eq. (7) that the constant $B$ and the coefficients $a_{n}$ may be expressed in terms of the unknown current density $j(r)$. Using the orthogonality properties of Bessel functions [7] it is found directly that

$$
B=\frac{2 \rho}{a^{2}} \int_{0}^{a} r j(r) d r
$$

and

$$
a_{n}=-\frac{2 \rho}{a^{2}\left[J_{0}\left(\beta_{n}\right)\right]^{2}} \int_{0}^{a} r j(r) J_{0}\left(\frac{\beta_{n} r}{a}\right) d r
$$

The integral in Eq. (8) is identical with $I / 2 \pi$ where $I$ is the total current through the constriction; the constant $B$ is thus immediately identified as $B=\rho I / \pi a^{2}$. Eq. (9) indicates that the problem of determining the coefficients $a_{n}$ may be reduced to the problem of finding the current density $j(r)$. The remainder of this section focuses on the procedure 
adopted for evaluating $j(r)$ explicitly and thus for solving the dual Dini series equations (5) and (6).

The procedure follows generally that developed by Sneddon and Srivastav [6]. The current density in the constriction is represented in terms of an unknown kernel function $h(t)$ as

$$
j(r)=-\rho^{-1} b^{2} \frac{1}{r} \frac{\partial}{\partial r} \int_{r / b}^{1} \frac{t h(t)}{\left(t^{2}-r^{2} / b^{2}\right)^{1 / 2}} d t, \quad r<b .
$$

Eqs. (8) and (9) may then be written in terms of $h(t)$ as

$$
\begin{gathered}
B=\frac{2 b^{2}}{a^{2}} \int_{0}^{1} h(t) d t \\
a_{n}=\frac{-2 b^{2}}{a^{2}\left[J_{0}\left(\beta_{n}\right)\right]^{2}} \int_{0}^{1} h(t) \cos \left(\frac{\beta_{n} b t}{a}\right) d t .
\end{gathered}
$$

The form of Eq. (10) was chosen so that the resulting representations (11) and (12) have the property that the Neumann boundary condition (6) for $r>b$ is identically satisfied independently of the particular form of the function $h(t)$. The explicit form of this function is thus determined by the Dirichlet condition (5) alone. Some insight into the physical significance of the representation (10) may be gained by examining the consequence of setting $h(t)$ equal to a constant $h_{0}$; in this case, substitution of the known value of $B$ into Eq. (11) yields $h_{0}=\rho I / 2 \pi b^{2}$. From Eq. (10) it then follows that

$$
j(r)=\frac{I}{2 \pi b^{2}} \frac{1}{\left(1-r^{2} / b^{2}\right)^{1 / 2}} .
$$

Eq. (13) reproduces the known limiting form for the current density as $b / a$ tends to zero [5]. The quantity $\left[h(t)-h_{0}\right]$ thus generates, via representation (10), the perturbing effects of the finite radial size of the cylinder on the current density distribution in the constriction. The representation (10) has the further attractive feature of manifestly preserving the singularity in the current density for $r=b$; this divergence is inherent in the formulation of the boundary-value problem with a sharp constriction edge.

The equation determining $h(t)$ is obtained by substituting the representation (12) into the Dirichlet condition (5). This yields immediately

$$
A-\frac{2 b^{2}}{a} \int_{0}^{1} h(t) \sum_{n=1}^{\infty} \frac{J_{0}\left(\beta_{n} r / a\right) \cos \left(\beta_{n} b t / a\right)}{\beta_{n}\left[J_{0}\left(\beta_{n}\right)\right]^{2}} d t=0
$$

where it has been assumed that the interchange in the order of summation and integration of infinite sums is valid (this assumption will be made throughout the remainder of the derivation). The sum in the integrand of Eq. (14) has the integral representation [6]

$$
\begin{aligned}
2 \sum_{n=1}^{\infty} \frac{J_{0}\left(\beta_{n} u\right) \cos \left(\beta_{n} v\right)}{\beta_{n}\left[J_{0}\left(\beta_{n}\right)\right]^{2}}=\theta(u-v)\left(u^{2}-v^{2}\right)^{-1 / 2}-2\left(1-v^{2}\right)^{1 / 2} \\
-\frac{2}{\pi} \int_{0}^{\infty} \frac{K_{1}(y)}{y I_{1}(y)} \cosh (v y)\left[2 I_{1}(y)-y I_{0}(u y)\right] d y
\end{aligned}
$$

where $\theta(x)$ is the conventional unit step function distribution and $I_{n}(y)$ and $K_{n}(y)$ are respectively the regular modified and singular modified Bessel functions of order $n$. Substi- 
tuting representation (15) into Eq. (14) yields the integral equation

$$
\int_{0}^{r / b} \frac{h(t)}{\left(r^{2} / b^{2}-t^{2}\right)^{1 / 2}} d t=\frac{A}{b}+\frac{b}{a} \int_{0}^{1} H(r, t) h(t) d t
$$

where

$$
H(r, t)=2\left(1-\frac{b^{2} t^{2}}{a^{2}}\right)^{1 / 2}+\frac{2}{\pi} \int_{0}^{\infty} \frac{K_{1}(y)}{y I_{1}(y)} \cosh \left(\frac{b t y}{a}\right)\left[2 I_{1}(y)-y I_{0}\left(\frac{r y}{a}\right)\right] d y .
$$

Eq. (16) may be reduced to a more familiar form by first solving it as though the right-hand side were a known function of $r$. This procedure yields [8]

$$
h(t)=\frac{2 A}{\pi b}+\int_{0}^{1} K(t, u) h(u) d u
$$

where $K(t, u)$ is defined by

$$
K(t, u)=\frac{2}{\pi} \frac{b}{a} \frac{d}{d t} \int_{0}^{t} \frac{v H(b v, u)}{\left(t^{2}-v^{2}\right)^{1 / 2}} d v .
$$

Eq. (18) has the standard form of a linear Fredholm integral equation of the second kind [8]. The kernel may be reduced [9] to the form

$$
K(t, u)=K_{0} \frac{b}{a}+\frac{4}{\pi^{2}} \frac{b}{a} \int_{0}^{\infty} \frac{K_{1}(y)}{I_{1}(y)}\left[1-\cosh \left(\frac{b u y}{a}\right) \cosh \left(\frac{b t y}{a}\right)\right] d y
$$

where

$$
K_{0}=\frac{4}{\pi}+\frac{4}{\pi^{2}} \int_{0}^{\infty} \frac{K_{1}(y)}{y I_{1}(y)}\left[2 I_{1}(y)-y\right] d y .
$$

Eq. (18) along with relations (20) and (21) now represent all the information required to generate $h(t)$ and thus permit an explicit evaluation of the current density $j(r)$ and of the coefficients $a_{n}$.

Since the function $K(t, u)$ is well-behaved in the range $0 \leq t, u \leq 1$ and vanishes as $b / a$ tends to zero, the classical iterative method of solution may be applied to Eq. (18) to determine $h(t)$. The solution is given by the Neumann series [8]

$$
h(t)=\frac{2 A}{\pi b} \sum_{n=0}^{\infty} h_{n}(t)
$$

where the functions $h_{n}(t)$ are determined by the iteration formula

$$
h_{n+1}(t)=\int_{0}^{1} K(t, u) h_{n}(u) d u
$$

with $h_{0}(t)=1$. In order to evaluate the functions $h_{n}(t)$ explicitly it will prove convenient to isolate the dependence on $t$ of both $h_{n}(t)$ and $K(t, u)$. Writing

$$
h_{n}(t)=\sum_{m=0}^{\infty} \alpha_{m}^{(n)} t^{2 m}, \quad K(t, u)=\sum_{m=0}^{\infty} \kappa_{m}(u) t^{2 m}
$$

in Eq. (23), where $\alpha_{m}^{(n)}$ and $\kappa_{m}(u)$ depend on the parameter $b / a$, leads to the recursion relation 


$$
\alpha_{m}^{(n)}=\sum_{l=0}^{\infty} \sigma_{m}^{l} \alpha_{l}^{(n-1)}
$$

where $\alpha_{m}^{(0)}=\delta_{m o}\left(\delta_{m n}\right.$ is the Kronecker symbol) and

$$
\sigma_{m}^{l}=\int_{0}^{1} u^{2 l} \kappa_{m}(u) d u
$$

The coefficients $\kappa_{m}(u)$ are obtained from Eq. (20) by expanding the hyperbolic cosine terms in the integral of that equation in a conventional Taylor series and integrating term by term. It is immediately found that

$$
\kappa_{m}(u)=K_{0} \frac{b}{a} \delta_{m o}-\frac{4}{\pi^{2}} \frac{b}{a} \sum_{\substack{n=0 \\ m+n \neq 0}}^{\infty} \frac{I_{2(n+m)}}{(2 m) !(2 n) !(2 n+2 m+1)}\left(\frac{b}{a}\right)^{2(n+m)} u^{2 n}
$$

where the integrals

$$
I_{2 n}=\int_{0}^{\infty} \frac{1}{\left[I_{1}(y)\right]^{2}} y^{2 n} d y
$$

for $n>0$ are well known and have been evaluated numerically by Smythe [10] for a large number of values of $n$. With the particular form of $\kappa_{m}(u)$ given by Eq. (28), Eq. (27) yields $\sigma_{m}^{l}$ directly as a series expansion in powers of $b / a$, i.e.,

$$
\sigma_{m}^{l}=-\sum_{n=0}^{\infty} \gamma_{m n}^{l}\left(\frac{b}{a}\right)^{2(m+n)+1}
$$

where

$$
\gamma_{m n}^{l}=-\frac{K_{0}}{(2 l+1)} \delta_{m o} \delta_{n o}+\frac{4}{\pi^{2}} \frac{I_{2(n+m)}}{(2 m) !(2 n) !(2 n+2 l+1)(2 n+2 m+1)} .
$$

In Eq. (31) the second term is zero when $m=n=0$. The dependence of $\alpha_{m}^{(n)}$ on $b / a$ can be similarly expressed. From Eqs. (26) and (30) it is found that

$$
\alpha_{m}^{(n)}=(-)^{n} \sum_{l=0}^{\infty} D_{m l}^{(n)}\left(\frac{b}{a}\right)^{2(l+m)+n}
$$

where the coefficients $D_{m l}^{(n)}$ are independent of $b / a$ and are determined recursively via

$$
D_{m l}^{(n)}=\sum_{k=0}^{l} \sum_{p=0}^{k} \gamma_{m p}^{l-k} D_{l-k, k-p}^{(n-1)}
$$

with the definition $D_{m l}^{(1)}=\gamma_{m l}^{0}$. The prescription (23) for generating the function $h(t)$ has thus been reduced to a procedure, given by Eqs. (24), (32) and (33), in which the $b / a$ dependence of $h(t)$ has been made explicit.

The solution for $h(t)$ obtained by including the first $N$ terms in the Neumann series (22) may be concisely expressed as

$$
h(t)=\frac{2 A}{\pi b}\left[1+\sum_{k=0}^{\infty} C_{k} t^{2 k}\right]
$$

where the coefficients $C_{k}$ are given by 


$$
C_{k}=\sum_{n=1}^{N} \alpha_{k}^{(r)}=\sum_{n=1}^{N} C_{k n}\left(\frac{b}{a}\right)^{2 k+n}
$$

with

$$
C_{k n}=(-)^{n} \sum_{l=1}^{p} D_{k,(p-l)}^{(2 l-1)}
$$

where $p$ is equal to $(n+1) / 2$ for $n$ odd and to $n / 2$ for $n$ even. The function $h(t)$ is thus conveniently given in terms of the coefficients $C_{k n}$. These coefficients are numerical constants which are independent of $b / a$ and which thus need be computed only once; according to formula (36) the coefficients are known exactly for $n \leq N$. The quantity $A$ is obtained by normalizing the solution (34) through the use of Eq. (11). This procedure yields directly $A=\rho I / 4 b S_{c}$ where

$$
S_{c}=1+\sum_{k=0}^{\infty} \frac{C_{k}}{(2 k+1)} .
$$

The derivation of the explicit expression (34) for $h(t)$ as generated through Eqs. (35) to (37) completes the formal stage of the calculation and permits the determination both of the current density $j(r)$ and of the potential distribution in the constricted cylinder. This evaluation is presented in the next section along with a calculation of the spreading resistance which represents one further quantity of physical interest.

It is clear from Eq. (34) that the accuracy with which the function $h(t)$ may be evaluated depends entirely on the accuracy with which the coefficients $C_{k}$ are known, i.e., on the number $N$ of terms included in expansion (35). In view of this it is instructive to examine the behavior of the coefficients $C_{k_{n}}$. Values for these coefficients computed from Eq. (36) are given in Table 1. It will be noted that these coefficients increase in magnitude with increasing index $n$; thus for large values of $b / a$ the first few terms in expansion (35) in fact increase in magnitude with $n$. For $b / a<1$, however, the damping effect of the factor $(b / a)^{n}$ eventually overcomes the increasing trend in $C_{k n}$ and successive terms yield decreasing contributions to the sum. As demonstrated in the next section, this behavior of $C_{k n}$ is essential for securing the correct limit for the spreading resistance when $b / a=1$. As a result of this behavior, however, convergence of expression (35) is slow as $b / a$ approaches unity. For any given value of $b / a<1$ the inaccuracy in the values of $C_{k}$ due to truncation of the sum (35) can be minimized by choosing $N$ sufficiently large. The coefficients $C_{k}$ themselves decrease in magnitude with increasing index $k$. These coefficients enter into the computation of the quantities of physical interest described below only through sums of the form (37) where each term involves the product of $C_{k}$ with a factor smaller than unity. Table 1, presented for $N=30$, contains a sufficient number of entries to ensure that the evaluation of these sums may be carried out with an accuracy of $1 \%$ at $b / a=0.8$ and within $0.1 \%$ for $b / a \leq 0.7$. Comparable accuracy for $b / a$ larger than 0.8 may be achieved by extending Table 1 through the use of Eqs. (33) and (36).

3. Results. Before proceeding with the evaluation of the distribution of electric potential it is instructive to consider two quantities of physical interest which may be evaluated directly from the solution for the function $h(t)$. The first of these quantities is the spreading resistance $R_{s}$ representing the contribution to the resistance of the cylinder arising from the constriction of current flow lines and defined by the expression 


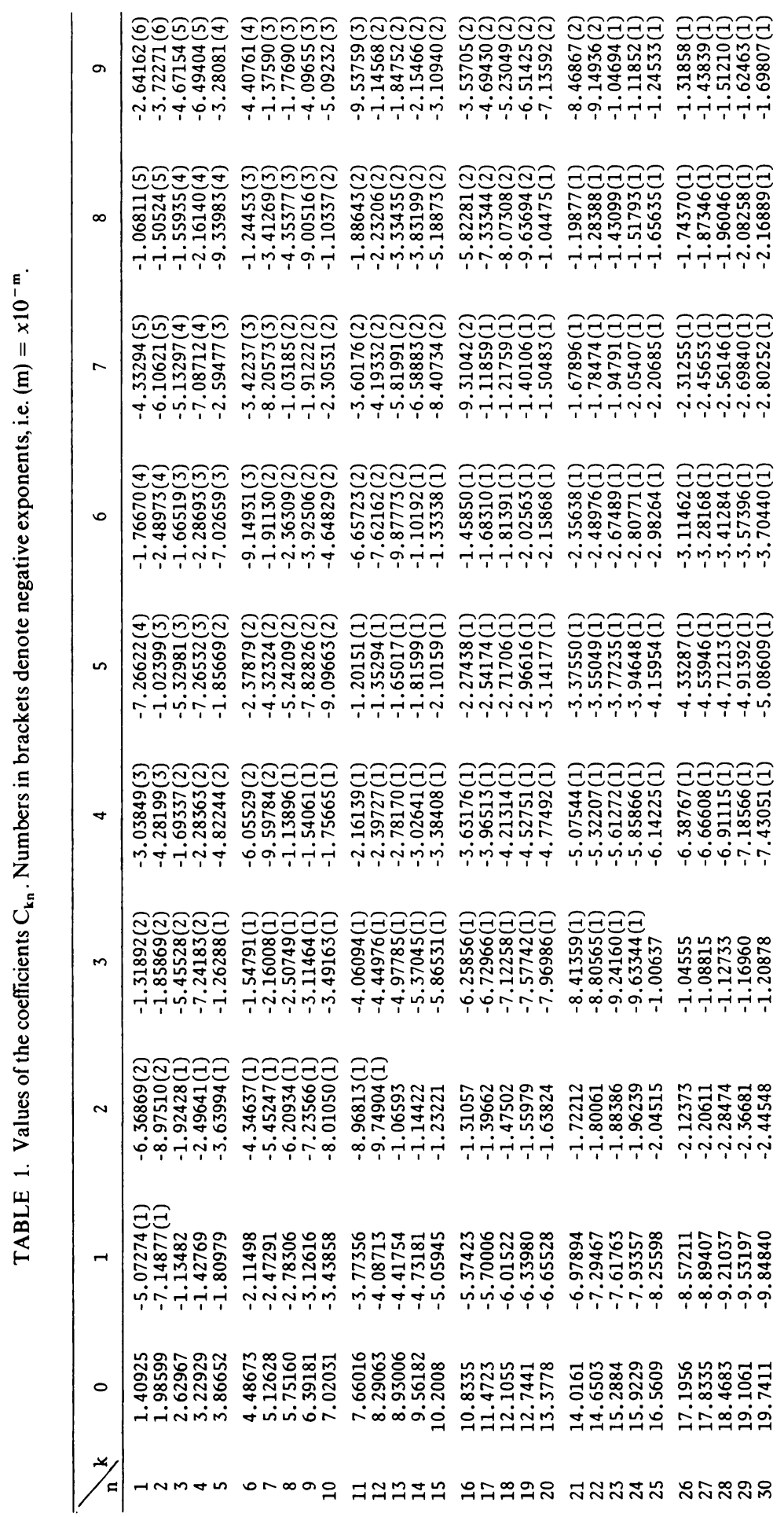




$$
R_{s}=\frac{V(a, l)-V(a,-l)}{I}-\frac{2 \rho l}{\pi a^{2}}
$$

where $l \gg a$ (i.e., far from the constriction). From Eq. (2) it is found immediately that $R_{s}=2 A / I$, where $A$ is calculated through Eq. (37), and hence it follows that

$$
R_{s}=\frac{\rho}{2 b}\left[1+\sum_{k=0}^{\infty} \frac{C_{k}}{2 k+1}\right]^{-1} \text {. }
$$

Expression (39) may be readily evaluated as a function of $b / a$ through Eq. (35) and the use of Table 1. This evaluation yields values of $R_{s}$ which coincide with those calculated from the expressions given by Roess [2] and Timsit [4] only for $b / a \lesssim 0.5$ where the results of these earlier treatments are expected to be accurate. For larger values of $b / a$, the results of the present calculation may be compared with the data of Clausing [3] who solved the contact resistance problem numerically for values of $b / a$ as large as 0.84 ; the comparison indicates agreement of the two sets of results to within the $1 \%$ level of accuracy quoted by Clausing for his analysis. It will be noted from the discussion of the preceding section that for $b / a=1$ the coefficients $C_{k}$ are unbounded and the sum in Eq. (39) diverges to yield $R_{s}=0$. The limiting value of $R_{s}$ for $b / a=1$ is thus given correctly by Eq. (39) since in this situation the cylinder is free from constriction and $R_{s}$ must indeed vanish.

A compact expression for the dependence of $R_{s}$ on $b / a$ as obtained from the present analysis may be generated by fitting a least-squares polynomial, with the constraints $R_{s}(b / a=0)=\rho / 2 b$ and $R_{s}(b / a=1)=0$, to values for $R_{s}$ computed from Eq. (39). This procedure leads to the expression

$$
R_{s}=\frac{\rho}{2 b}\left[1-1.41581\left(\frac{b}{a}\right)+0.06322\left(\frac{b}{a}\right)^{2}+0.15261\left(\frac{b}{a}\right)^{3}+0.19998\left(\frac{b}{a}\right)^{4}\right]
$$

which reproduces the results computed from the exact expression (39) to within $0.1 \%$ for $b / a \leq 0.7$ and to within a maximum deviation of $1 \%$ at $b / a=0.8$. The error decreases identically to zero at the constraint endpoint $b / a=1$. It is worth noting that Eq. (40) is similar in form to the expression obtained by Roess [2] and Timsit [4].

The second quantity which may be evaluated at this stage is the current density distribution $j(r)$; this evaluation yields some further insight into the effect of the finite diameter of the constricted cylinder on current flow near the constriction. Using expression (34) for $h(t)$, $j(r)$ is found directly from Eq. (10) as

$$
j(r)=\frac{I}{2 \pi b^{2}\left(1-r^{2} / b^{2}\right)^{1 / 2}} \frac{1}{S_{c}}\left[1+\sum_{k=0}^{\infty} \eta_{k}\left(1-\frac{r^{2}}{b^{2}}\right)^{k}\right]
$$

where

$$
\eta_{k}=\frac{(-)^{k}(2 k+1)}{(2 k+1) ! !} \sum_{l=k}^{\infty} \frac{(2 l) ! !}{(2 l-2 k) ! !} C_{l} .
$$

The current density $j(r)$ can be immediately evaluated for any value of $b / a$ through the use of Eq. (35) and the results in Table 1. For $b / a$ approaching zero the correct limiting form (13) is recovered by noting that $\eta_{k}=0$ and $S_{c}=1$ when $b / a=0$. The variation of $j(r)$ with $r$ 


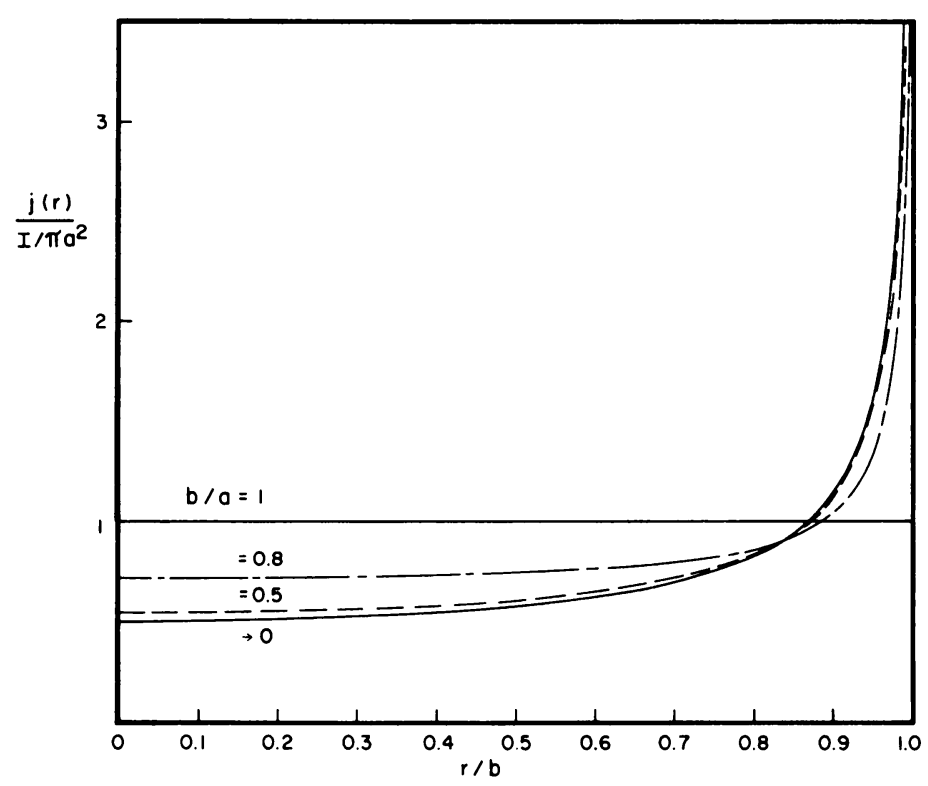

FIG. 2. Constriction current density $j(r)$ as a function of $r / b$.

for several values of $b / a$ is illustrated in Fig. 2. It is interesting to note that the deviation of the actual distribution $j(r)$ from the limiting form as $b / a \rightarrow 0$ becomes significant only for values of $b / a$ approaching unity.

The determination of the potential distribution proceeds directly from expression (34) for $h(t)$. Using Eqs. (2) and (12), the solution for the potential can be written in the form

$$
V(r, z)=\frac{1}{2} I R_{s}+B z-\frac{\rho I}{\pi b} V_{0}(r, z)
$$

where

$$
V_{0}(r, z)=\frac{b}{a} \sum_{n=1}^{\infty} \frac{J_{0}\left(\beta_{n} r / a\right) \exp \left(-\beta_{n} z / a\right)}{\beta_{n}\left[\left(J_{0}\left(\beta_{n}\right)\right]^{2}\right.} \frac{1}{S_{c}} \sum_{k=0}^{\infty}\left(C_{k}+\delta_{k o}\right) \int_{0}^{1} t^{2 k} \cos \left(\frac{\beta_{n} b t}{a}\right) d t .
$$

This expression is exact and rigorously valid for all values of $b / a<1$. However, the particular form given by Eq. (44) is not the most convenient for purposes of evaluation. In particular, for $z$ close to zero the oscillatory behavior of the Bessel function and the loss of damping by the exponential factor in the first sum of Eq. (44) conspire to yield extremely slow convergence of the series. In this case several hundred terms of the sum are required for a satisfactory evaluation of the potential. Clearly a more manageable expression for the potential is desirable. This situation was encountered in a previous publication [4] and is addressed in the next section along the general lines followed in that earlier work.

4. Evaluation of the potential. In order to transform Eq. (44) into a form which may be readily evaluated it proves convenient to write that equation in the form

$$
V_{0}(r, z)=\frac{1}{S_{c}} \sum_{k=0}^{\infty}\left(\delta_{k o}+C_{k}\right) \int_{0}^{1} t^{2 k} \frac{d}{d t} S(r, z, t) d t
$$


where

$$
S(r, z, t)=\sum_{n=1}^{\infty} \frac{f\left(r, z, t ; \beta_{n}\right)}{\beta_{n}\left[J_{0}\left(\beta_{n}\right)\right]^{2}}
$$

with

$$
f(r, z, t ; \beta)=\frac{1}{\beta} \sin \left(\beta \frac{b}{a} t\right) J_{0}\left(\beta \frac{r}{a}\right) \exp \left(-\beta \frac{z}{a}\right) .
$$

Using standard techniques from the theory of residues [7] it is found that sum (46) has the contour integral representation

$$
S(r, z, t)=\frac{1}{2} G(r, z, t ; \infty)-\frac{1}{2 \pi i} \int_{\varepsilon-i \infty}^{\varepsilon+i \infty} \frac{G(r, z, t ; \beta)}{\beta\left[J_{1}(\beta)\right]^{2}} d \beta
$$

where $0<\varepsilon<\beta_{1}$ and

$$
G(r, z, t ; \beta)=\int_{0}^{\beta} f(r, z, t ; \omega) d \omega .
$$

In order to evaluate expression (48) an explicit expression for $G(r, z, t ; \beta)$ must be obtained. This is readily accomplished for finite $\beta$ by representing $f(r, z, t ; \omega)$ from Eq. (47) as a product of the conventional Taylor series expansion for the sine, Bessel and exponential functions and integrating term by term in Eq. (49). This procedure leads immediately to an expression for $G(r, z, t ; \beta)$ as a product of power series of each of its arguments. Substituting this form into Eq. (48) yields $S(r, z, t)=G(r, z, t ; \infty) / 2-S_{1}(r, z, t)$ where

$$
S_{1}(r, z, t)=\sum_{l, m, n=0}^{\infty} \frac{(-)^{l+m+n} B_{2 l+2 n+m}}{2^{2 l}(l !)^{2} m !(2 n+1) !(2 l+2 n+m+1)}\left(\frac{r}{a}\right)^{2 l}\left(\frac{z}{a}\right)^{m}\left(\frac{b}{a} t\right)^{2 n+1}
$$

with

$$
B_{k}=\frac{1}{2 \pi i} \int_{\varepsilon-i \infty}^{\varepsilon+i \infty} \frac{\beta^{k}}{\left[J_{1}(\beta)\right]^{2}} d \beta .
$$

The integrals $B_{0}$ and $B_{1}$ have the values $M_{0} / 2 \pi$ and 2 respectively where $M_{0}=6.95437$ [4]. From the relation $J_{1}(i \beta)=i I_{1}(\beta)$ it follows immediately that $\beta_{2 k}$ is identical with $(-)^{(k+1)} I_{2 k} / \pi$ where $I_{2 k}$ is defined by Eq. (29). For odd $k>1, B_{k}$ vanishes from symmetry considerations. Thus beyond the first power, only even powers of $z$ contribute in Eq. (50). On rearranging the summations in that equation and collecting terms homogeneous in $r$ and $z, S_{1}$ can be expressed as

$$
\begin{aligned}
S_{1}(r, z, t)=\left(\frac{b}{a} t\right)\left(\frac{M_{0}}{2 \pi}-\frac{z}{a}\right)-\frac{1}{\pi} \sum_{l, n=0}^{\infty} \frac{I_{2(l+n)}}{(2 l) !(2 n+1) !(2 l+2 n+1)} \\
\times\left(\frac{b}{a} t\right)^{2 n+1}\left(\frac{r^{2}}{a^{2}}+\frac{z^{2}}{a^{2}}\right)^{l} P_{l}^{(-1 / 2,0)}\left(\frac{r^{2}-z^{2}}{r^{2}+z^{2}}\right)
\end{aligned}
$$

where $P_{l}^{(\alpha, \beta)}(x)$ is the orthogonal Jacobi polynomial of order $l$ with parameters $\alpha$ and $\beta$ [11]. The reduction of Eq. (44) can now be completed. From Eqs. (45), (48) and (52) it follows that the potential distribution is given by Eq. (43) with 


$$
\begin{array}{r}
V_{0}(r, z)=\frac{1}{2 S_{c}}\left(1+\sum_{k=0}^{\infty} C_{k}\right) G(r, z, 1 ; \infty)-\frac{1}{S_{c}} \sum_{k=1}^{\infty} k C_{k} \int_{0}^{1} t^{2 k-1} G(r, z, t ; \infty) d t \\
\quad-\frac{b}{a}\left(\frac{M_{0}}{2 \pi}-\frac{z}{a}\right)+\frac{1}{\pi S_{c}} \sum_{l=0}^{\infty} F_{l}\left(\frac{r^{2}}{a^{2}}+\frac{z^{2}}{a^{2}}\right)^{l} P_{l}^{(-1 / 2,0)}\left(\frac{r^{2}-z^{2}}{r^{2}+z^{2}}\right)
\end{array}
$$

where $G(r, z, t ; \infty)$, defined by Eq. (49), is given by [9]

$$
G(r, z, t ; \infty)=\arcsin \left\{\frac{2 b t}{\left[z^{2}+(b t+r)^{2}\right]^{1 / 2}+\left[z^{2}+(b t-r)^{2}\right]^{1 / 2}}\right\}
$$

and

$$
F_{l}=\sum_{n=0}^{\infty} \frac{I_{2(l+n)}}{(2 l) !(2 n) !(2 l+2 n+1)}\left(\frac{b}{a}\right)^{2 n+1} \sum_{k=0}^{\infty} \frac{\left(\delta_{k o}+C_{k}\right)}{2 k+2 n+1} .
$$

Expression (53) for $V_{0}(r, z)$ provides considerable computation advantage over the original expression (44) for the evaluation of the potential. One noteworthy feature of expression (53) is the ease with which the limiting form of $V_{0}(r, z)$ as $b / a$ approaches zero is obtained; in this situation all terms except that involving $G(r, z, 1 ; \infty)$ vanish and the factor $\left(1+\Sigma C_{k}\right) / S_{c}$ in this term approaches unity. The closed form given by Eq. (54) for the remaining component $G(r, z, 1 ; \infty)$ is particularly easy to evaluate and represents in fact the exact solution for the case of a constriction in an infinitely large cylinder [5]. For $b / a \neq 0$ the evaluation of expression (53) remains straightforward; the integrals over $G(r, z, t ; \infty)$ can be readily computed numerically and the Jacobi polynomials can be generated without difficulty from well-known recurrence relations [11]. However, the number of terms required from each of the summation components in Eq. (53) to achieve a selected accuracy in $V_{0}(r, z)$ depends on the magnitudes of $b / a, r / a$ and $z / a$. A general account of the effort required to evaluate the potential is best summarized as follows. For $b / a$ appreciably smaller than unity $(b / a<0.2)$ the terms additional to the arcsine component may be viewed as perturbations on the potential arising from the distant cylinder walls and very few terms ( $\leq 4$ terms) are required from each of the summations to compute $V_{0}(r, z)$ with high accuracy. Although these perturbations become large when $b / a$ is an appreciable fraction of unity, an accurate evaluation of the potential (subject to the limitations of Table 1) may still be obtained with fewer than ten terms from each of the summations in Eq. (53) when $b / a \leq 0.8$, provided $0 \leq r / a, z / a \leq 0.5$. Under these conditions the full advantage of expression (53) over the form (44) is in fact realized. It is only when $r / a$ and $z / a$ approach unity that caution must be exercised in evaluating the summations to ensure that a sufficient number of terms are included to achieve the accuracy desired.

Finally, it is clear that the procedure outlined in this section ceases to be valid when $z / a \geq 1$; in this case the potential must be evaluated through recourse to the original expression (44).

[1] W. R. Smythe, J. Appl. Phys. 23, 170 (1952)

\section{REFERENCES}

[2] L. C. Roess, Theory of spreading conductance, Appendix A of an unpublished report of the Beacon Laboratories of Texas Company, Beacon, New York

[3] A. M. Clausing, University of Illinois Department of Mechanical and Industrial Engineering, ME Technical Report 242-2, Urbana, 1965 
[4] R. S. Timsit, J. Phys. D, Appl. Phys. 10, 2011 (1977)

[5] F. Llewellyn-Jones, The physics of electrical contacts, Oxford Press, 1957

[6] I. N. Sneddon and R. P. Srivastav, Proc. Roy. Soc. Edin. A66, 150 (1964)

[7] G. N. Watson, A treatise on the theory of Bessel functions, Cambridge University Press, 1966

[8] F. G. Tricomi, Integral equations, Interscience, New York, 1957

[9] I. S. Gradshtyn and I. M. Ryzhik, Table of integrals, series and products, Academic Press, New York, 1965

[10] W. R. Smythe, Phys. Fluids Vol. 7, 633 (1964)

[11] M. Abramowitz and I. A. Stegun, Handbook of mathematical functions, Dover, New York, 1972 